\section{Salud y Pobreza en Chile de Post-Transición: ¿Qué nos dicen los indicadores de pobreza sobre la salud de la población?}

\author{
LEANDRO GONZÁLEZ,a, CARLOS VILLALOBOS²,b
}

\section{Degree of association between healthcare indicators and poverty measures in Chile over the period 1992-2017}

Background: More than half of the worlds's population is deprived of essential healthcare services. In consideration of this, the World Health Organization introduced the concept of Social Determinants of Health to improve the awareness of this problem. Aim: To investigate and compare the pertinence of monetary and multidimensional measures of poverty as indirect measures of health status. Material and Methods: Three indices were used: the Historic Multidimensional Poverty Index (HMPI), calculated using Alkire-Foster method; health deprivations associated with the HMPI; and households in conditions of monetary poverty. The poverty identification outcomes for the three indices mentioned were all estimated using data from the Chilean national socioeconomic survey CASEN for the period 1992-2017. Results: First, independently of how poverty is measured (monetarily or multidimensionally), the degree to which households living in poverty conditions are simultaneously suffering health deprivations steadily decreased during the period 1992-2017. Second, the association between multidimensional poverty and health deprivations is stronger than the association between health deprivations and monetary poverty. Conclusions: Poverty calculated on the basis of income alone is an inadequate predictor of health deprivations; multidimensional poverty performs better. However, poverty and health indicators have become progressively less associated. Therefore, it becomes necessary either to adapt the health indicators included in Multidimensional Poverty Index to the current health challenges or to implement a Multidimensional Health Deprivation Index, with a view to improving the integration of health within the current social policy framework.

(Rev Med Chile 2021; 149: 779-789)

Key words: Poverty; Public Health Systems Research; Public Policy; Quality Indicators, Health Care; Social Protection.

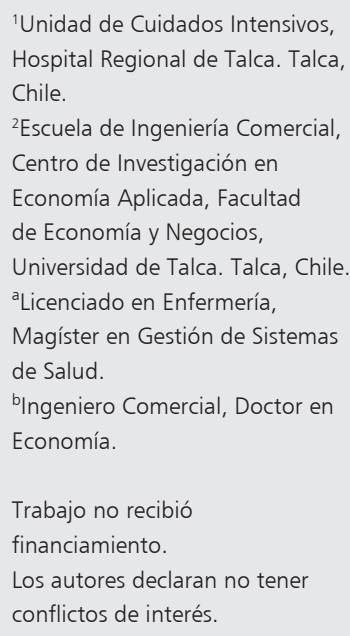

Recibido el 30 de septiembre de 2020, aceptado el 3 de junio de 2021.

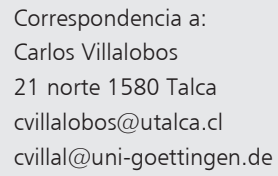


en salud ${ }^{2-4}$, las DSS además han sido reconocidas en los Objetivos de Desarrollo Sostenible (ODS).

Esta evidencia es prueba del vínculo que se ha asumido entre la condición de pobreza monetaria y las carencias en salud. Por una parte, la falta de acceso a la salud, y también carencias en educación, entorpecen la acumulación de capital humano contribuyendo al establecimiento de círculos de pobreza crónica ${ }^{5}$. Por otra parte, y especialmente en una economía en donde la provisión de salud se ha entregado en parte importante al mercado, la falta de ingresos afecta negativamente las posibilidades de las familias de llevar vidas saludables al dificultar su acceso a una buena alimentación, agua potable, a servicios sanitarios adecuados, o bien a combustibles limpios que no contaminan el aire al interior de las viviendas, entre otras ${ }^{6}$.

En este contexto de mercantilización de la salud, el gasto de bolsillo en salud aumentó 39,5\% per cápita entre 1997 y $2007^{7}$. En 2012 este gasto representó en promedio 5,2\% del gasto total de los hogares, y lo más preocupante es que para dos de cada 5 hogares de menores recursos, este gasto tuvo una connotación catastrófica ${ }^{8}$.

Podríamos criticar que el gasto en salud no sería el producto de un proceso de maximización del bienestar que otorgue la importancia que los individuos libremente dan a su salud. Si la proporción del gasto en salud es baja, entre otras razones, puede deberse a que el sistema de salud y la educación enfatizan la medicina preventiva por sobre la curativa, no implicando esto que los individuos tengan una menor valoración por su salud. Así, los axiomas clásicos de la maximización de utilidad de la teoría económica clásica pueden ser inadecuados y, en consecuencia, más gasto en salud y más prestaciones curativas no estarían necesariamente asociadas a mayores niveles de bienestar. Por esta razón, el transitar desde la medicina curativa a una preventiva introduce necesariamente distorsiones en la línea de pobreza monetaria que, desde un punto de vista metodológico, no pueden ser controladas de una manera sencilla.

En consecuencia, la idea de que las carencias en salud se resolverían con una mayor dotación de ingresos de las familias es cuestionable. De hecho, la evidencia empírica muestra un desacople entre la disponibilidad de los recursos y el nivel de carencias en salud. Alkire y Santos enumeran los factores que contribuyen a este desacople?
Entre estos factores se encuentran los distintos patrones de consumo de los hogares, como estos se distribuyen entre los miembros del hogar, los distintos precios que éstos enfrentan, la forma en como los hogares transforman sus recursos en bienestar, y el hecho de que muchos de los bienes que facilitan la obtención de logros en salud no se obtienen necesaria y exclusivamente a través de los mercados.

La hipótesis de desacople entre los ingresos y la salud cuenta con cierta evidencia empírica que la soporta. Dreze y Sen muestran que a pesar de que India tiene un mayor Producto Interno Bruto que los países del África Sub-Sahariana, estos últimos países logran mejores indicadores en mortalidad infantil, inmunización y expectativa de vida ${ }^{10}$. Por su parte, Bourguignon et al. no encuentra asociación entre la reducción de la pobreza monetaria y un descenso en carencias no monetarias, incluidas las de la salud, exceptuando la desnutrición ${ }^{11,12}$. Klasen muestra que las carencias en salud no están estrechamente vinculadas con la pobreza en Sudáfrica $^{13}$. En el caso de los hogares rurales en China, Wang et al. muestra que la correlación existente entre la falta de acceso a un seguro de salud es prácticamente indistinguible entre hogares que sufren pobreza monetaria de aquellos que no la sufren ${ }^{5}$. Bourguignon y Chakravarty muestran que mejoras en indicadores de bienestar no monetario (como salud y educación) requieren de una mejora en la provisión de bienes y servicios públicos ${ }^{6}$. Así, desde el punto de vista de la oferta de salud, carencias o limitaciones de los servicios de salud (que predominantemente afectan con mayor intensidad a los sectores rurales) disminuyen los niveles de salud de la población, aun cuando esta tenga los recursos monetarios suficientes para demandar los servicios de salud que requiere ${ }^{13}$.

A pesar de la evidencia presentada anteriormente, en el caso de Chile la evidencia es mixta. Olavarría utilizando la encuesta CASEN entre 1987 y 2000 encuentra que los pobres monetarios tienen serias dificultades para acceder a la atención de salud cuando la necesitan, y cuando la obtienen, acceden a ella de manera tardía ${ }^{14}$. Bedregal et al. encuentra que las personas en condición de extrema pobreza desconfían del sistema de salud y perciben obstáculos para acceder a el ${ }^{15}$. Vega et al. muestra que, durante los 90s, las mujeres tenían serias desventajas de acceso a salud en comparación con los hombres, ya que a pesar de que los 
salarios de ellas eran en promedio 30\% inferiores al de los hombres, las mujeres usan los servicios de salud con mayor intensidad (1,5 veces más) ${ }^{16}$.

En contraposición con estos hallazgos y utilizando la encuesta CASEN del año 2000, Subramanian et al. encuentran que el ingreso individual no explica diferencias en la percepción de la propia salud agregada a nivel comunal ${ }^{17}$.

Es importante señalar que desde ya hace dos décadas han existido notorios avances en la forma de conceptualizar y medir la pobreza. Con mucha fuerza se ha adoptado en Chile desde 2013 el enfoque de las capacidades (capabilities) de $\mathrm{Sen}^{18}$, que ha sido implementado exitosamente mediante el Índice de Pobreza Multidimensional (MPI por sus siglas en inglés). El MPI captura el bienestar en salud mediante la medición de los logros en nutrición, acceso y cobertura de los sistemas de salud.

Se abre así la oportunidad de investigar la hipótesis de desacople entre la pobreza multidimensional y las carencias en salud. El único estudio que aborda esta hipótesis es el realizado por Cabieses et al. utilizando los datos de la encuesta CASEN de $2013^{19}$. Los autores encuentran que la condición de pobreza multidimensional afecta negativamente la salud auto-percibida de la población.

En este estudio, investigamos la hipótesis de desacople de las carencias en salud y la pobreza (monetaria y multidimensional) con una amplia perspectiva temporal (1992-2017). Para ello, primero se describen las trayectorias de pobreza monetaria y multidimensional, y de las carencias en salud que se han vivido en Chile durante los últimos 25 años. Luego, se provee inédita información sobre el grado de asociación en la identificación del pobre (monetario y multidimensional) y el carente en salud. Este grado de asociación permite evaluar la pertinencia de ambas métricas de pobreza como mediciones indirectas del bienestar en salud, y abre la interrogante sobre el cómo se inserta a la salud dentro de las medidas del bienestar agregadas para su articulación en función de la política social en Chile. Vale mencionar que este trabajo viene a ampliar y complementar la interesante discusión sobre la interrelación entre salud y pobreza en Chile ${ }^{14-17,19-21}$.

La sección 2 presenta brevemente las metodologías empleadas en la medición de la pobreza monetaria y multidimensional, y luego se describe la medida de asociación que se emplea para medir el grado en que los distintos índices e indicadores identifican simultáneamente a los individuos en su condición de pobreza y carencias en salud. Los resultados se muestran en la sección 3. Finalmente, la conclusión se desarrolla en la sección 4.

\section{Materiales y Métodos}

Para estudiar el nivel de asociación en la identificación del pobre monetario y multidimensional y las carencias en salud, se requiere estimar el Índice de Pobreza Multidimensional Histórico (HMPI) ${ }^{22}$ utilizando el método $\mathrm{AF}^{23}$, las carencias de salud asociadas al HMPI, y la identificación de los hogares en condición de pobreza monetaria según la metodología FTG ${ }^{24}$. El HMPI considera cuatro dimensiones y 12 indicadores, teniendo cada uno de ellos un peso equivalente (Tabla 1).

El HMPI es una adaptación del MPI de 4 dimensiones de 2013, debido a que, éste no es susceptible de ser calculado consistentemente para el período 1992-2011 (Figura 1). En Chile, un hogar es identificado como pobre multidimensional cuando la suma relativa de sus carencias alcanza el 25\%, mientras que la condición de pobreza por ingreso se otorga a los hogares que no pueden acceder a una canasta básica de bienes y servicios. La canasta mínima congruente con un adecuado estándar de vida es capturada por las distintas líneas oficiales de pobreza monetaria. La estimación de los índices e indicadores anteriormente señalados se realiza en base a los microdatos de las diferentes encuestas de caracterización económica CASEN para los años 1992, 1994, 1996, 1998, 2000, 2003, 2006, 2009, 2011, 2013, 2015, у 2017.

En relación con la medición del nivel de asociación en la identificación de los pobres/carentes, la Tabla 2 muestra la distribución conjunta existente entre dos carencias en dos dimensiones de bienestar $j$ y $j^{\prime}$. Si las medidas de carencia no son independientes, y al menos una de las razones de conteo $\left[\mathbb{P}_{\mathbf{0 0}}^{j j^{\prime}}, \mathbb{P}_{\mathbf{1 1}}^{j j^{\prime}}, \mathbb{P}_{\mathbf{1 0}}^{i j^{\prime}}, \mathbb{P}_{\mathbf{0 1}}^{j j^{\prime}}\right]$ es distinta de cero, la medida de redundancia $R^{0}$ mide el grado de asociación o simultaneidad en la identificación de las carencias de las dimensiones $j$ y $j$ ' en la población ${ }^{25}$. Se puede entonces definir la medida de redundancia $R^{0}$ como el grado en que las personas son simultáneamente carentes en ambos indicado- 
Tabla 1. Indicadores de salud para la medición de la pobreza multidimensional histórica

\begin{tabular}{|c|c|}
\hline Indicador & Definición: personas que viven en ... \\
\hline \multicolumn{2}{|r|}{ Educación } \\
\hline Asistencia & $\begin{array}{l}\text { Hogares donde al menos un niño o adolescente de 4-18 años no asiste al colegio y quien } \\
\text { aún no se ha graduado (después de completar } 12 \text { años de escolaridad). Cambios res- } \\
\text { pecto del indicador de 2013: Se excluye la información sobre la ausencia permanente } \\
\text { de aquellos 4-26 debido a que las encuestas CASEN 1992, 1994, 1996, 1998, } 2000 \text { y } \\
2003 \text { no la contienen. }\end{array}$ \\
\hline Rezago escolar & $\begin{array}{l}\text { Hogares donde al menos una persona menor de } 21 \text { años cursando educación primaria/ } \\
\text { secundaria que se encuentre con al menos dos años de rezago bajo su correspondiente } \\
\text { nivel. No hay cambios respecto del indicador de } 2013 \text {. }\end{array}$ \\
\hline Escolaridad & $\begin{array}{l}\text { Hogares donde al menos una persona se encuentre bajo el mínimo legal para su cohorte. } \\
\text { Nacidos entre 1920-1924. } 4 \text { años de escolaridad; nacidos entre 1930-1965. } 6 \text { años de } \\
\text { escolaridad; nacidos entre } 1966 \text {-2002. } 8 \text { años de escolaridad. Desde el } 2003 \text { en adelante } \\
\text { el mínimo legal fue de } 12 \text { años. No hay cambios respecto del indicador de } 2013 .\end{array}$ \\
\hline
\end{tabular}

\section{Salud}

Malnutrición Hogares donde al menos un niño/niña menor de 6 años es clasificado como desnutrido en niños (o en riesgo de desnutrición) u obeso según evaluación nutricional en control de salud. Cambios respecto del indicador de 2013: en 1992, 1994, 1996, 1998, 2000, 2003, y 2006, las variables relevantes se refieren a niños de 0-5 años (a diferencia del resto que considera niños de 0-6 años).

Seguro de Hogares donde se encuentre al menos una persona no adscrita a un sistema previsional salud de salud. Cambios respecto del indicador de 2013: La información sobre seguros de salud complementarios se excluye porque en 1992, 1994, 1996, 1998, 2000, 2003, 2006, y 2009, la encuesta CASEN es provista.

Acceso a salud Hogares en que al menos una mujer de 21 años no se haya realizado el examen Papanicolau en los últimos 3 años o bien no tenga conocimiento del examen. Cambios respecto del indicador de 2013: La definición del MPI 2013 considera como hogares carentes si hay al menos un miembro del hogar que ha sufrido un problema de salud durante los últimos 3 meses y no recibió tratamiento, o si en los últimos 12 meses, esta persona lo recibió y no fue cubierta por el programa (AUGE-GES). Esta compleja definición es imposible de adoptar debido a que los programas relevantes fueron recién creados en 2005 y el tiempo retrospectivo de referencia cambia constante desde la encuesta CASEN 2000 en adelante.

\section{Trabajo y seguridad social}

Ocupación Hogares con al menos un miembro de 18 años o más que se encuentra desempleado y

Seguridad social

Jubilaciones

Hacinamiento Hogares con al menos un miembro de 15 años o más que no se encuentra contribuyendo al sistema de pensiones y no ha estado en educación terciaria. No hay cambios respecto del indicador de 2013.

Hogares donde al menos un miembro femenino de 60 años o más. o masculino de 65 años o más. no está recibiendo una pensión de retiro. No hay cambios respecto del indicador de 2013.

\section{Vivienda}

Hogares en los cuales el promedio de personas compartiendo una habitación es más alto que 2,5. No hay cambios respecto del indicador de 2013.

Estado de la Viviendas en los cuales el piso, techo y muros se encuentran en mal estado u hogares vivienda hechos de materiales de baja calidad. No hay cambios respecto del indicador de 2013.

Servicios Hogares sin suministro de agua al interior de la vivienda (área urbana) o acceso a agua Básicos segura (área rural) o carecen de servicio sanitario o fosa séptica (en áreas rurales y
Ponderación urbanas). No hay cambios respecto del indicador de 2013.

Fuente: Klasen y Villalobos (2020). 


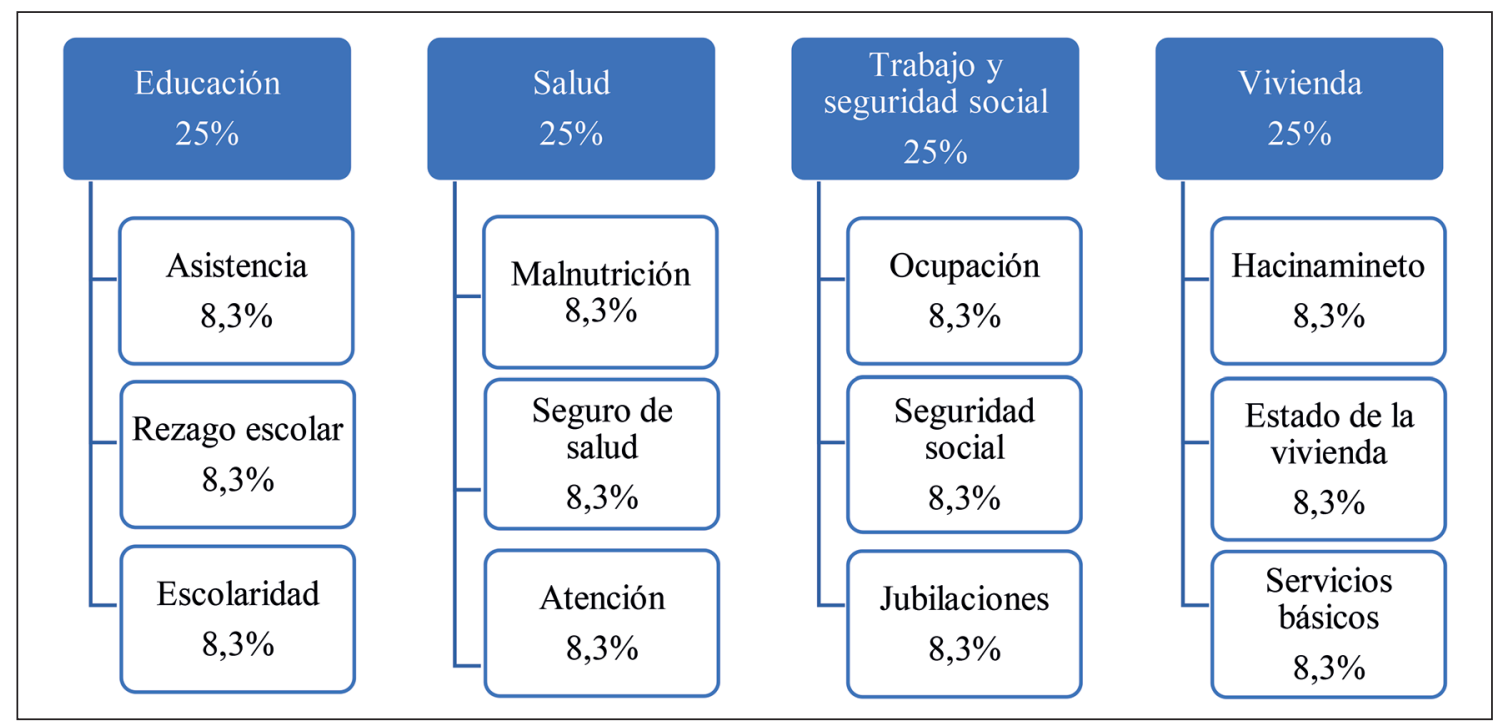

Figura 1. Ponderación de indicadores y dimensiones para la medición de la pobreza multidimensional. Fuente: Ministerio de Desarrollo Social (2013).

Tabla 2. Tabla de contingencia de carencias en dos dimensiones

\begin{tabular}{|llccc|}
\hline & & & Dimensión $\boldsymbol{J}^{\prime}$ \\
& & No carente & Carente & Total \\
\hline \multirow{2}{*}{ Dimensión J } & Carente & $\mathbb{P}_{00}^{j j^{\prime}}$ & $\mathbb{P}_{01}^{j j^{\prime}}$ & $\mathbb{P}_{0+}^{j}$ \\
& Total & $\mathbb{P}_{10}^{j j^{\prime}}$ & $\mathbb{P}_{11}^{j j^{\prime}}$ & $\mathbb{P}_{1+}^{j}$ \\
& & $\mathbb{P}_{+0}^{j^{\prime}}$ & $\mathbb{P}_{+1}^{j^{\prime}}$ & 1 \\
\hline
\end{tabular}

Fuente Alkire et al. (2015).

res (carente en indicador de salud y condición de pobreza monetaria/multidimensional). A mayor $R^{0}$, mayor es el grado de asociación entre las medidas de bienestar.

$R^{0}$ es definida como:

$$
R^{0}=\frac{\mathbb{P}_{11}^{j j^{\prime}}}{\min \left\{\mathbb{P}_{+\mathbf{1}}^{j^{\prime}}, \mathbb{P}_{\mathbf{1}}^{j}\right\}} \quad 0 \leq R^{0} \leq 1
$$

\section{Resultados}

En la Tabla 3 se presentan las estimaciones de pobreza multidimensional y sus carencias constituyentes, incluidas las de salud, las estimaciones de pobreza monetaria y las medidas de redundancia asociadas. Todos los indicadores de bienestar han mejorado de manera sustancial entre 1992 y 2017.

En relación con el HMPI, siendo su valor en 1992 de 0,201 , este ha progresado hasta un nivel de 0,094 en 2017. Esta significativa caída es producto de una sustancial reducción en el número de personas que viven en situación de pobreza multidimensional (55,1\% de la población en 1992 y 30,9\% en 2017), y de una reducción en la intensidad con la que esta población sufre carencias acumuladas (casi 37\% en 1992 y $31 \%$ en 2017). En cuanto a la pobreza monetaria, ésta ha caído desde casi 33\% en 1992 a 8,6\% en 2017. La evolución es también favorable cuando se observa la caída en la pobreza extrema desde 9\% en 1992 a 2,3\% en 2017.

Con relación al porcentaje de la población 


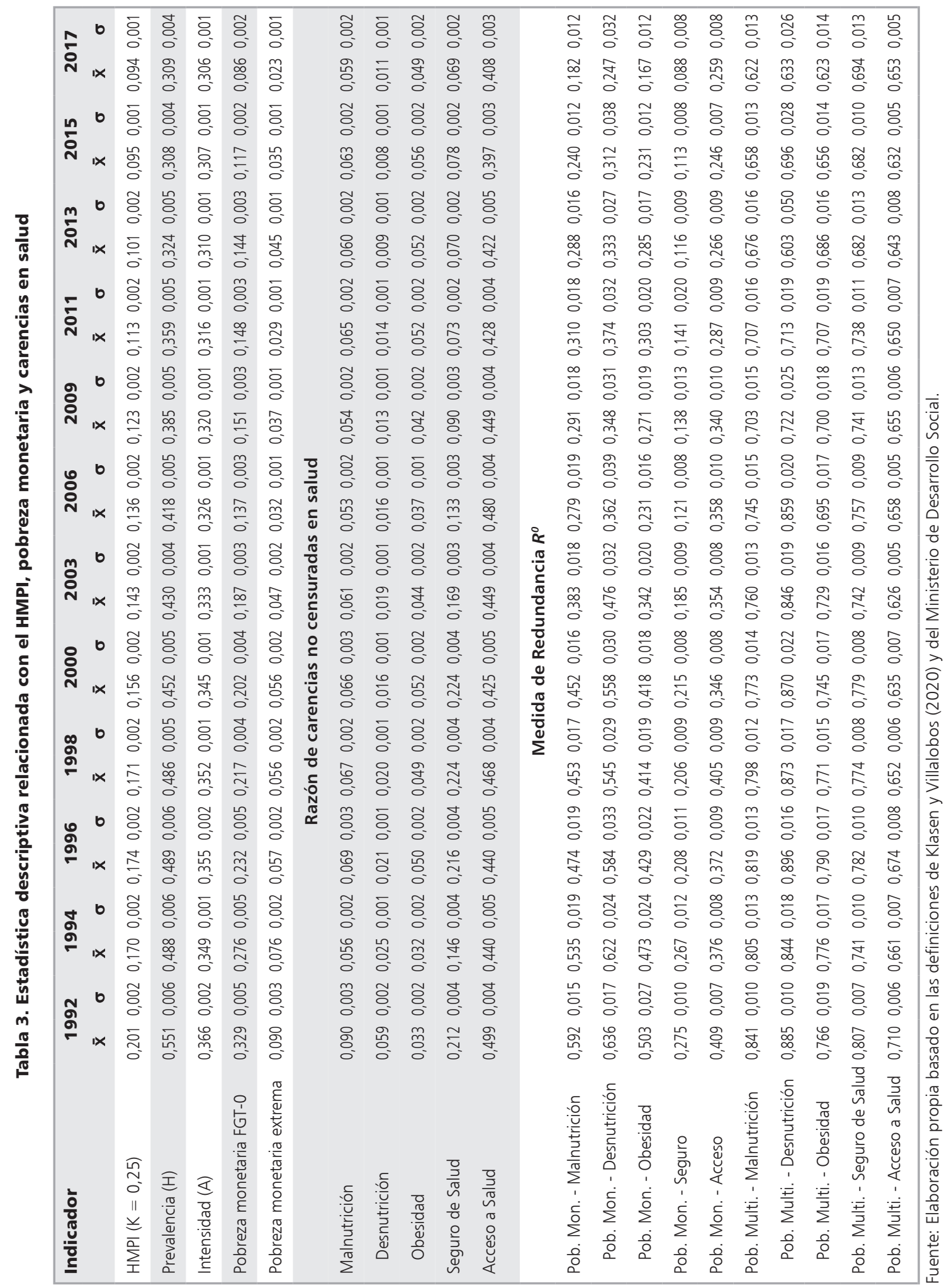


clasificada en situación de malnutrición, esta se ha reducido desde 9\% en 1992 a 5,9\% en 2017. Esta evolución conjuga una positiva evolución en la desnutrición (que desciende desde 5,9\% en 1992 a $1,1 \%$ en 2017) con un desempeño no tan favorable en los niveles de obesidad (que crecen desde $3,3 \%$ en 1992 a 4,9\% en 2017). El porcentaje de la población clasificada con carencia en su acceso a salud muestra una disminución desde $50 \%$ en 1992 a 41\% en 2017. Finalmente, en el indicador de cobertura de salud, el porcentaje de la población clasificada con carencia disminuye desde $21,2 \%$ en 1992 a 6,9\% en 2017 (Figuras 2 y 3).
Las disímiles trayectorias en el progreso observado en los índices agregados de pobreza y en los indicadores de nutrición, acceso a la salud y cobertura de seguro de salud, podrían evidenciar que estas no están ni estrecha ni simétricamente acopladas. Para dar luz a este asunto, a continuación, se presenta la evolución de la medida de redundancia $R^{0}$.

En términos generales, se observa una disminución sostenida del $R^{0}$ entre 1992 y 2017 entre los indicadores de nutrición (malnutrición, desnutrición y obesidad) y ambos indicadores de pobreza (monetaria y multidimensional). Estos resultados nos muestran que casi $60 \%$ de
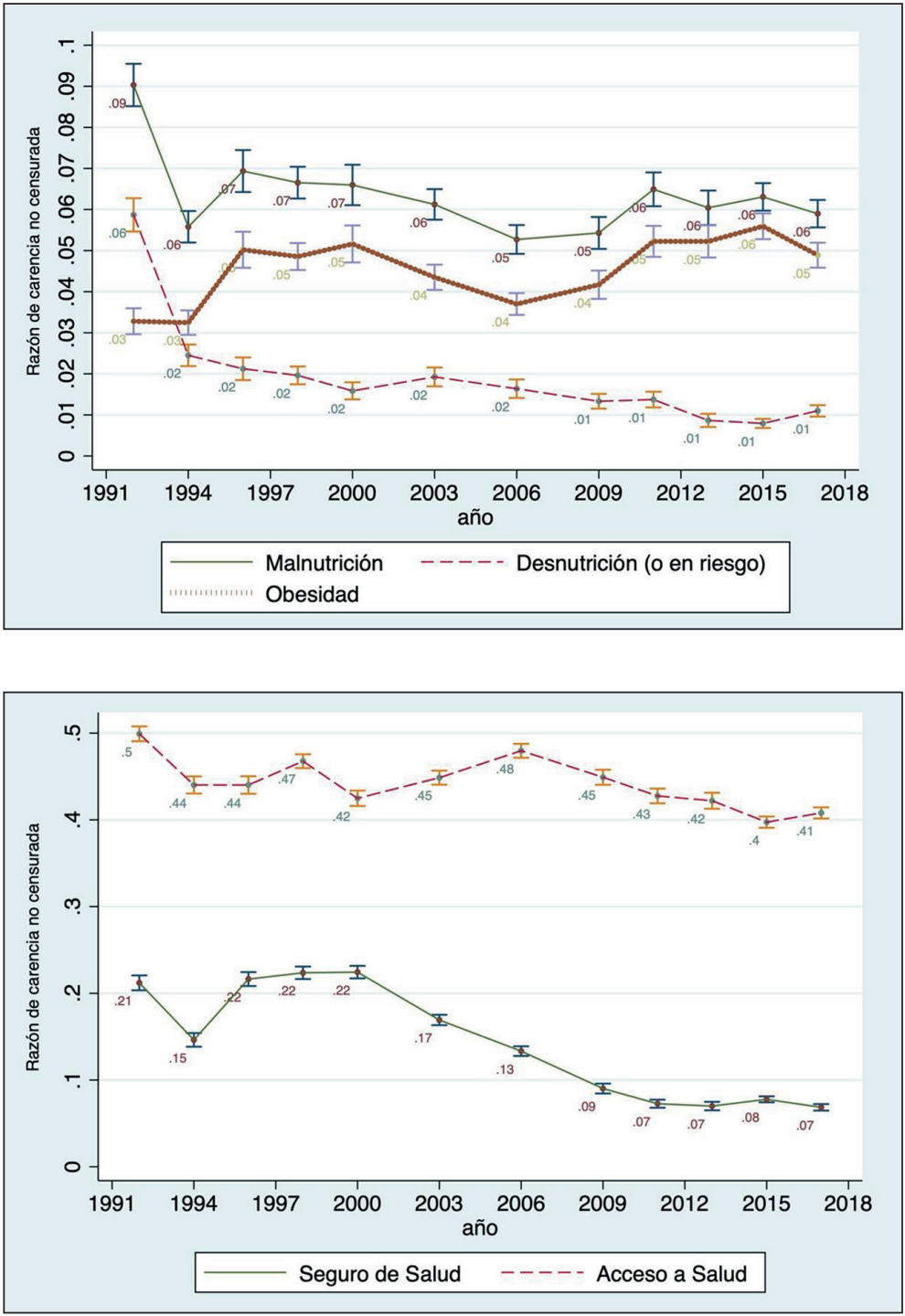

Figura 2. Razón de carencias no censuradas para el indicador malnutrición. Fuente: Elaboración propia. Nota: Intervalos de confianza de $95 \%$.
Figura 3. Razón de carencias no censuradas para seguro de salud y acceso a salud. Fuente: Elaboración propia. Nota: Intervalos de confianza de $95 \%$. 
la población identificada como carente en malnutrición fue simultáneamente pobre monetario en el año 1992, cifra que llega a $18 \%$ en 2017 . Se concluye entonces que la carencia en nutrición y el fenómeno de la pobreza monetaria se dan cada vez con menor grado de simultaneidad (Figura 4).

La redundancia entre la pobreza monetaria y la carencia en cobertura de salud muestra caída pronunciada, desde $27,5 \%$ en 1992 a 8,8\% en 2017. La simultaneidad entre la carencia en acceso a salud y pobreza monetaria también se reduce desde $41 \%$ en 1992 a 26\% en 2017 (Figura 5).
La redundancia entre los indicadores de malnutrición y la pobreza multidimensional también es decreciente. Mientras el $R^{0}$ alcanzó casi $84 \%$ en 1992, en 2017 su valor fue de 62,2\% que es mucho mayor al valor obtenido para la redundancia contra la pobreza monetaria que en 2017 llegaba a $18,2 \%$. El $R^{0}$ de los indicadores de obesidad y desnutrición disminuyen en el tiempo hasta 2009, año en que estos indicadores convergen. El $R^{0}$ pasa de $76,6 \%$ y $88,5 \%$ en 1992 respectivamente a $62,3 \%$ y $63,3 \%$ en 2017 (Figura 6).

La misma dinámica ocurre con la asociación
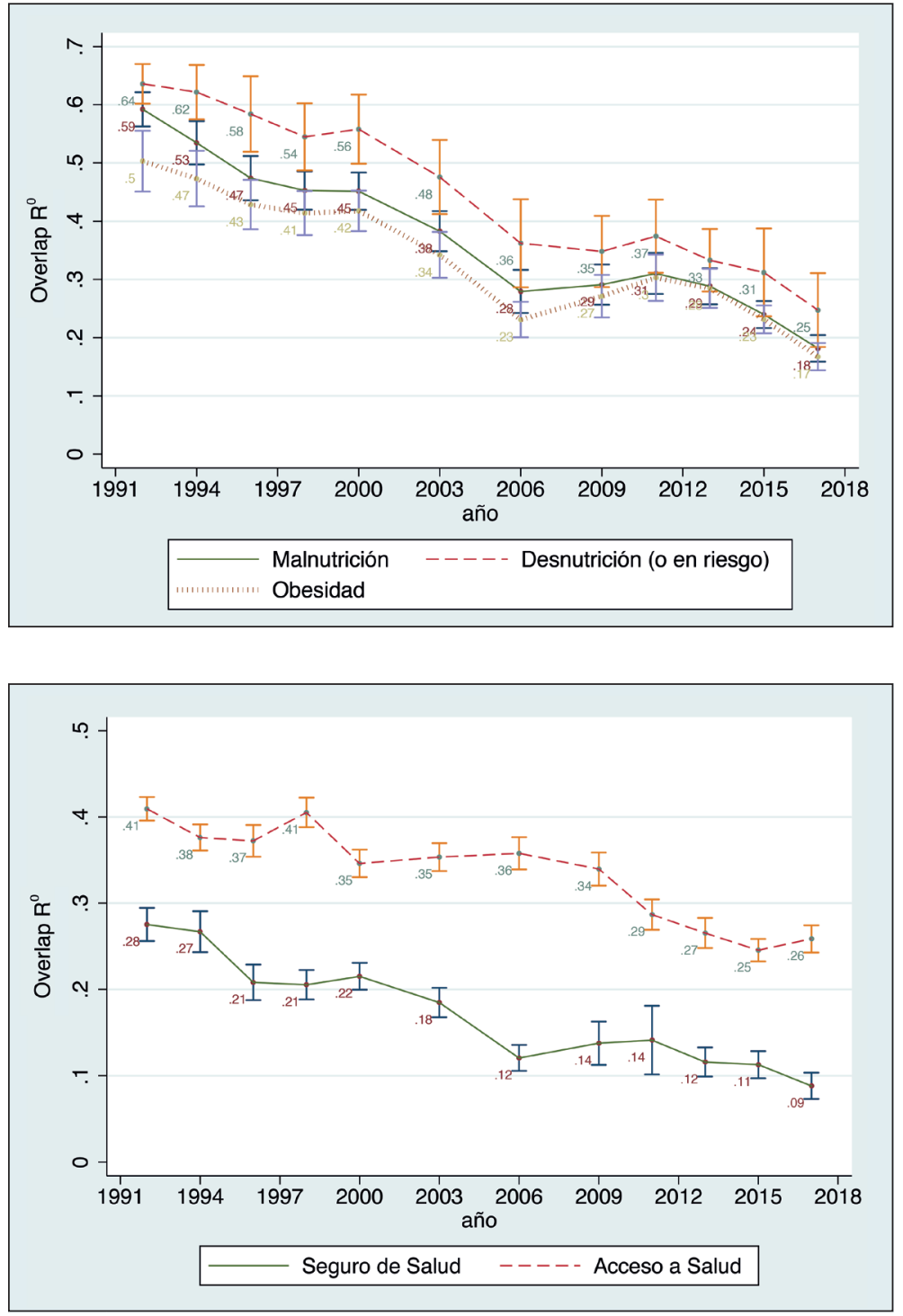

Figura 4. Medida de redundancia $R^{0}$ entre la pobreza monetaria e indicadores de malnutrición. Fuente: Elaboración propia. Nota: Intervalos de confianza de $95 \%$.

Figura 5. Medida de redundancia $R^{0}$ entre la pobreza monetaria e indicadores seguro de salud y acceso a salud. Fuente: Elaboración propia. Nota: Intervalos de confianza de $95 \%$. 
entre la pobreza multidimensional y los indicadores de carencia de seguro y acceso a salud. En 1992 el $R^{0}$ fue de $80,7 \%$ y $71 \%$ en carencia de seguro y acceso a salud respectivamente. En 2017, las medidas llegan a $69,4 \%$ y $65,3 \%$ respectivamente (Figura 7).

De estos resultados son dos las lecciones importantes que se pueden obtener. Primero, independientemente de la forma en cómo la pobreza sea medida, los niveles de asociación de estas medidas con los indicadores disponibles de la dimensión salud han decrecido sostenidamente en el tiempo. Segundo, tal y como es esperado, se comprueba que la medida de pobreza multidimensional tiene un nivel de asociación significativamente mayor (aunque igualmente decreciente) con los indicadores de salud, que el que estos mismos indicadores tienen con la medida de pobreza monetaria.

\section{Conclusión y Discusión}

Nuestra investigación muestra como durante el período 1992-2017 ha existido una notoria y
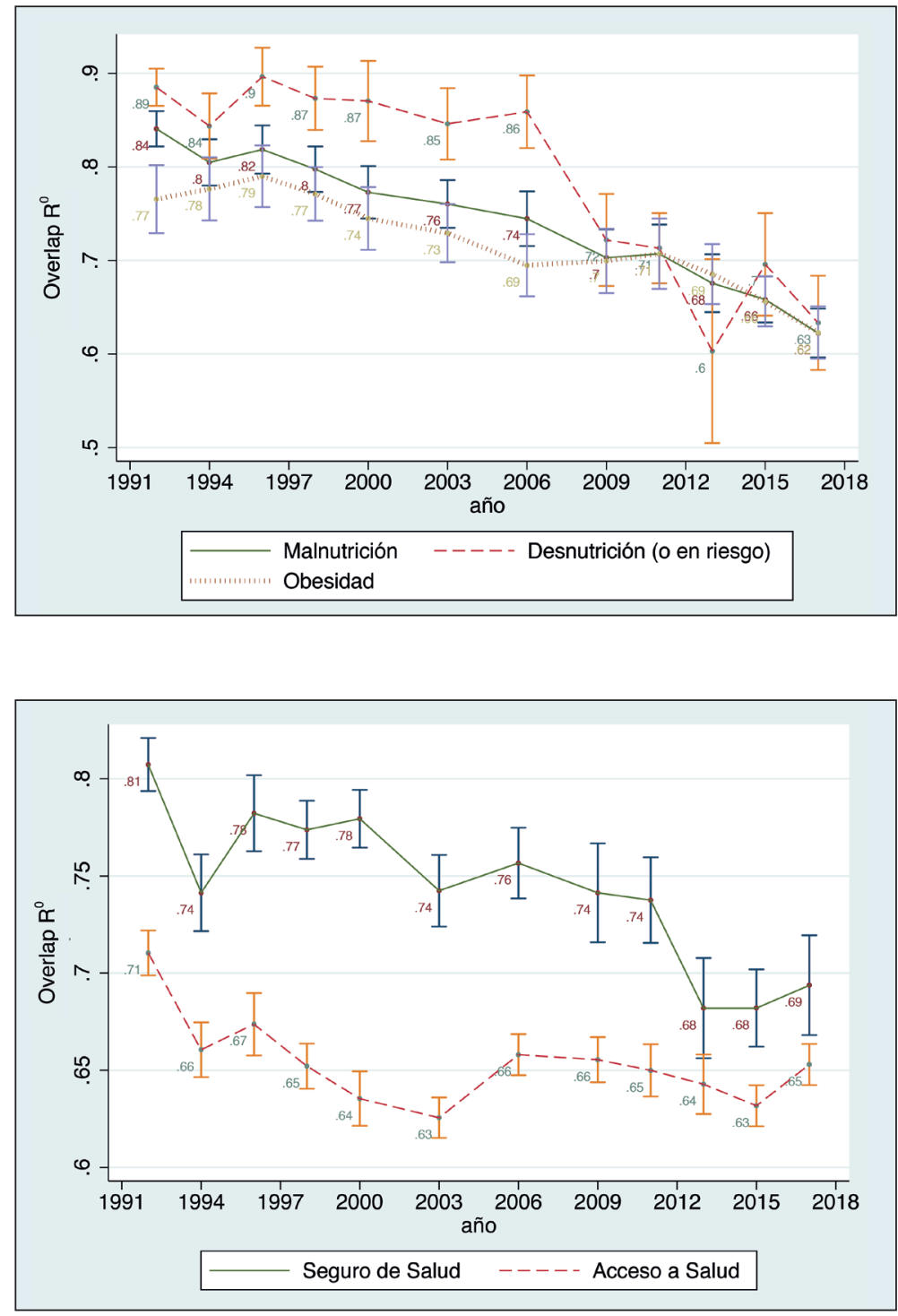

Figura 6. Medida de redundancia $R^{0}$ entre la pobreza multidimensional e indicadores de malnutrición. Fuente: Elaboración propia. Nota: Intervalos de confianza de $95 \%$.
Figura 7. Medida de Redundancia $R^{0}$ entre Pobreza Multidimensional e indicadores de seguro y acceso a salud. Fuente: Elaboracion Propia. Nota: Intervalos de confianza de $95 \%$. 
significativa reducción de los niveles de pobreza (independientemente de cómo esta sea medida), y de las carencias de salud. Este desarrollo, no se restringe necesariamente a los indicadores en salud utilizados y calculados en base a los microdatos de la encuesta CASEN, sino que probablemente responde a las mejoras subyacentes en salud que han tenido lugar en Chile durante las últimas tres décadas.

Esta mejoría general se explica por el progreso económico experimentado durante las últimas décadas, siendo también consecuencia de las sólidas políticas públicas en salud como el Programa $\mathrm{Na}$ cional de Inmunizaciones, el Programa Nacional de Alimentación Complementaria (PNAC), el Programa Nacional de Salud de la Infancia, y el Programa de Alimentación Complementaria del Adulto Mayor (PACAM), entre otros. El progreso en salud ha tenido lugar en países como México que coincide con Chile en aspectos como la liberalización comercial y el progreso tecnológico, pero no necesariamente en sus políticas públicas en salud ${ }^{26}$. Por otra parte, el desarrollo del sistema mixto de seguros de salud ha contribuido, por una parte, a promover la cobertura universal de salud, pero por otra, ha traído como consecuencia un creciente gasto de bolsillo de familias en salud, aumentando así el riesgo de padecer pobreza monetaria entre los hogares con menores ingresos.

La evidencia presentada en este trabajo es concluyente en afirmar que hoy, la medida de pobreza monetaria no captura adecuadamente el estado de carencias en salud de la población (habiéndolas capturado en mayor grado durante la década de los noventa). En contrapartida, y tal como es esperado, la pobreza multidimensional HMPI empleada en este estudio se asocia en mucho mayor grado con las carencias en salud de la población aproximada por los indicadores de salud disponibles. Hoy en día, más de la mitad de los individuos identificados con carencias en salud son pobres multidimensionales. No obstante, preocupante es el hecho de que el nivel de asociación también ha decrecido significativamente durante las últimas décadas.

Una vez descartado el indicador de pobreza monetaria para que nos informe de los progresos en salud, se abren interrogantes respecto a la necesidad de adaptar el MPI a los nuevos desafíos de la salud en Chile, de modo que las mediciones de pobreza multidimensional sigan reflejando avances y retrocesos en la salud de la población. De lo contrario, se podría dar el sinsentido de que, en un futuro no muy lejano, los pobres multidimensionales sean en baja proporción carentes en salud y viceversa.

En un enfoque alternativo y complementario, se abre la alternativa de discutir la estimación $R^{0}$ de un Índice de Carencia Multidimensional de Salud (ICMS) en donde se integren, además de factores socioeconómicos relevantes para la salud, sub-dimensiones de esta, tales como la salud mental y adicciones, violencia, autocuidado y prevención, y medidas de nutrición más complejas, que por ejemplo incluyan elementos como la diversidad dietética, déficits de nutrientes, o que ponderen en mayor medida flagelos modernos como el de la obesidad (no solo infantil), entre otras. Vale decir que todas estas dimensiones antes mencionadas son actualmente excluidas de las estimaciones oficiales de pobreza multidimensional de 4 y 5 dimensiones ${ }^{27}$.

La respuesta a cuál de los dos enfoques es preferible adoptar para que las medidas de pobreza sigan siendo buenas aproximaciones del estado de salud general de la población no es de fácil respuesta. Sin embargo, debe avanzarse en el debate al respecto, en especial cuando se busca que el indicador de pobreza multidimensional pueda capturar los progresos en el avance de la medicina preventiva por sobre la curativa, el rol del autocuidado en salud y el nivel de éxito de las políticas públicas de salud. En definitiva, este estudio pretende estimular la reflexión sobre los indicadores de salud y el cómo estos debiesen estar integrados a la política social vía los indicadores agregados de pobreza.

\section{Referencias}

1. Marmot M, Friel S, Bell R, Houweling TA, Taylor S. Closing the gap in a generation: health equity through action on the social determinants of health. The Lancet 2008; 372 (9650): 1661-9.

2. Cerf ME. Sustainable Development Goal Integration, Interdependence, and Implementation: the Environment-Economic-Health Nexus and Universal Health Coverage. Glob Challenges 2019; 3 (9): 1900021.

3. World Health Organization. Tracking Universal Health Coverage. First global monitoring report. 2015. Disponible en: https:/www.who.int/health_financing/ documents/tracking-uhc/en/. 
4. Winslow CEA. The cost of sickness and the price of health. Bulletin of the World Health Organization 2006; 84 (2): 153-8. Disponible en: https://apps.who.int/iris/ handle/10665/269572.

5. Wang X, Feng H, Xia Q, Alkire S. On the relationship between Income Poverty and Multidimensional Poverty in China. OPHI Working Paper 2016; 101. Oxford Poverty \& Human Development Initiative. Disponible en: https://www.ophi.org.uk/wp-content/uploads/OPHIWP101_1.pdf.

6. Bourguignon F, Chakravarty SR. The Measurement of Multidimensional Poverty. En: Chakravarty S. Editores. Poverty, Social Exclusion and Stochastic Dominance. Singapore, Springer; 2019. p. 83-107. https://doi. org/10.1007/978-981-13-3432-0_7.

7. Cid-Pedraza C, Prieto Toledo L. El gasto de bolsillo en salud: El caso de Chile, 1997 y 2007. Revista Panamericana de Salud Publica 2012; 31 (4): 310-6.

8. Bruzzo S, Henríquez J, Velasco C. Radiografía del gasto de bolsillo en salud en Chile: una mirada desagregada. Puntos de Referencia. 2018; 478. Disponible en: https://www.cepchile.cl/cep/site/ docs/20180316/20180316105839/pder478_jhenriquez_ sbruzzo_cvelasco.pdf.

9. Alkire S, Santos ME. Measuring Acute Poverty in the Developing World: Robustness and Scope of the Multidimensional Poverty Index. World Dev 2014; 59: 25174.

10. Drèze J, Sen A. An uncertain glory: India and its contradictions. Princeton, Princeton University Press. 2013. doi: 10.2307/j.ctt32bcbm.

11. Bourguignon F, Bénassy-Quéré A, Dercon S, Estache A, Gunning JW, Kanbur R, et al. The millennium development goals: an assessment. In Kanbur R, Spence M. Editores, Equity and growth in a globalising World. Washington DC, World Bank; 2010. p 17-39.

12. Klasen S. Poverty, undernutrition, and child mortality: Some inter-regional puzzles and their implicationsfor research and policy. J Econ Inequal 2008; 6: 89-115. https://doi.org/10.1007/s10888-007-9056-x.

13. Klasen S. Measuring poverty and deprivation in South Africa. Rev Income Wealth 2000; 46 (1)33-58. https:// doi.org/10.1111/j.1475-4991.2000.tb00390.x.

14. Olavarría Gambi M. Acceso a la salud en Chile. Acta Bioeth 2005; 11 (1): 47-64. http://dx.doi.org/10.4067/ S1726-569X2005000100006.

15. Bedregal P, Zavala C, Atria J, Núñez G, Pinto MJ, Valdés S. Acceso a redes sociales y de salud de población en extrema pobreza. Rev Med Chile 2009; 137 (6): 753-8. http://dx.doi.org/10.4067/S003498872009000600004 .
16. Vega MJ, Bedregal GP, Jadue HL, Delgado BI. Equidad de género en el acceso a la atención de salud en Chile. Rev Med Chile 2003; 131 (6): 669-78. http://dx.doi. org/10.4067/S0034-98872003000600012.

17. Subramanian V, Delgado BI, Jadue HL, Kawachi I, Vega MJ. Inequidad de ingreso y autopercepción de salud: Un análisis desde la perspectiva contextual en las comunas chilenas. Rev Med Chile 2003; 131 (3): 321-30. http:// dx.doi.org/10.4067/S0034-98872003000300012.

18. Sen A. Commodities and Capabilities. Amsterdam: North-Holland; 1985.

19. Cabieses B, Pedrero V, Oyarte M, Sirlopu D, Alfaro J, Bernales M, et al. Pobreza Multidimensional, Salud Auto-Percibida y Acceso a Salud en Chile: Generando Conocimiento Crítico para la Acción en Red en Salud Pública. Santiago de Chile: Universidad del Desarrollo. 2017. Disponible en: https://repositorio.udd.cl/handle/11447/1633.

20. Melo Z, Araya R, Valdés C. Acceso a diagnóstico y tratamiento para depresión según posición social en países seleccionados de Latinoamérica. Rev Chil Salud Pública 2010; 14 (2-3): 311-36.

21. Jiménez S, Barriga $O$, Salazar A. Inequidad en el acceso a salud en Chile: estudio multifactorial basado en la Encuesta CASEN del año 2013. Rev Chil Salud Pública 2018; 22 (1): 31-40.

22. Klasen S, Villalobos C. Diverging identification of the poor: A non-random process. Chile 1992-2017. World Dev 2020; 130: 104944. https://doi.org/10.1016/j.worlddev.2020.104944.

23. Alkire S, Foster J. Counting and multidimensional poverty measurement. J Public Econ. 2011; 95 (7-8): 476-87. https://doi.org/10.1016/j.jpubeco.2010.11.006.

24. Foster J, Greer J, Thorbecke E. A Class of Decomposable Poverty Measures. Econometrica 1984; 52 (3): 761-66. https://doi.org/10.2307/1913475.

25. Alkire S, Foster J, Seth S, Santos ME, Roche JM, Ballon P. Multidimensional Poverty Measurement and Analysis. Chapter 7. Oxford University Press. 2015. https:// doi.org/10.1093/acprof:oso/9780199689491.003.0007.

26. Villalobos Barría C, Klasen S, Vollmer S. The Distribution Dynamics of Human Development in Mexico 1990-2010. Rev Income Wealth 2016; 62: 47-67. https:// doi.org/10.1111/roiw.12220.

27. Ministerio de Desarrollo Social. Metodología de medición de pobreza multidimensional con entorno y redes. Serie Documentos Metodológicos No 32, División Observatorio Social. Santiago de Chile; 2016. Disponible en: http://observatorio.ministeriodesarrollosocial.gob. cl/storage/docs/pobreza/Metodologia_de_Medicion_ de_Pobreza_Multidimensional.pdf. 